\title{
Mineralocorticoid Receptor Antagonism and Aldosterone Synthesis Inhibition Do Not Improve Glomerulosclerosis and Renal Interstitial Fibrosis in a Model of Chronic Kidney Allograft Injury
}

\author{
Tobias Lahmer ${ }^{\mathrm{a}}$ Rob Hermans ${ }^{\mathrm{c}}$ Christoph Schmaderer ${ }^{\mathrm{a}}$ Jianxing Chang ${ }^{\mathrm{a}}$ \\ Konrad Stock ${ }^{\mathrm{a}}$ Jens Lutz ${ }^{\mathrm{b}} \quad$ Uwe Heemann $^{\mathrm{a}}$ Marcus Baumann ${ }^{\mathrm{a}}$ \\ ${ }^{a}$ Department of Nephrology, Klinikum rechts der Isar, Technische Universität München, München, ${ }^{b}$ Department of \\ Nephrology, Universitätsklinik Mainz, Mainz, Germany; ${ }^{\mathrm{C}}$ Department of Pharmacology and Toxicology, Maastricht \\ University, Maastricht, The Netherlands
}

\section{Key Words}

Aldosterone $\cdot$ Chronic allograft injury · Fadrozole •

Spironolactone

\begin{abstract}
Chronic allograft injury (CAI) is a major cause of late graft failure with a multifactorial pathogenesis; however, in different experiments an inhibition of the renin-angiotensin system by angiotensin-converting enzyme inhibitors and angiotensin II type 1 receptor blockers ameliorated the progression of chronic renal disease. Different concepts supposed that aldosterone is involved in development and/ or progression of renal diseases via interaction with a nonepithelial mineralocorticoid receptor (MR), e.g. reducing neointima formation. Our examinations therefore targeted on the effects of the aldosterone synthase inhibitor fadrozole and the MR antagonist spironolactone compared to vehicle in an established rat model of CAI. In our model of CAI, neither the aldosterone biosynthesis inhibitor nor a direct MR blockade had a positive effect on renal CAl in rats. Fadrozole- and spironolactone-treated animals demonstrated a higher proteinuria value, pathologically elevated potassi-
\end{abstract}

um values, higher tubulointerstitial damage and markedly increased heart weight/body weight as compared to vehicle. Our observations also suggest that inhibition of the MR or the biosynthesis itself had a bad influence on the amount of sclerotic glomeruli and tubulointerstitial damage. The positive effects of inhibition of aldosterone as described in cardiac models could not yet be detected in kidney recipients.

Copyright $\odot 2012$ S. Karger AG, Basel

\section{Introduction}

Chronic allograft injury (CAI) remains the main reason for long-term renal transplant failure [1]. CAI is a multifactorial process and is caused by both alloantigendependent and alloantigen-independent factors. Shortterm graft survival is based on the one hand on new immunosuppressive agents and new operation and preservation techniques. On the other hand, research has focused on improving HLA mismatches reducing acute rejection which limits the development and progression of CAI, respectively. However, improvements in long- 
term graft survival could not be obtained. Histologically CAI is characterized by glomerulopathy, tubular atrophy, interstitial fibrosis and vasculopathy [2]. The initial graft damage is mediated by ischemia and reperfusion [3]. Other reasons are surgical trauma and alloantigen-related factors such as acute rejection which influence the cause and progression of CAI. CAI is based on different pathogenetic factors which cannot be influenced effectively at the moment [3]. However, in different experiments an inhibition of the renin-angiotensin system by angiotensin-converting enzyme inhibitors and angiotensin II type 1 receptor blockers ameliorates the progression of chronic renal disease [4].

Another approach in CAI could be to block the mineralocorticoid receptor (MR), the receptor for the hormone aldosterone. Beyond the classic effects of promoting renal sodium retention and potassium/magnesium loss, it is well documented that aldosterone for example causes myocardial and perivascular fibrosis which can lead to vascular damage, endothelial dysfunction and decreased vascular compliance [5]. Therefore, the renin-angiotensin-aldosterone system plays a major role in the development of for example hypertension and heart failure, and is therefore a key target for therapeutic interventions [5].

Considering the success of MR antagonists and the established role of aldosterone in various diseases, for example cardiac fibrosis, cardiac remodeling, chronic heart failure or the progression on chronic kidney disease, different examinations were started to search for drug candidates that interfere with aldosterone in a manner blocking its receptor or decreasing aldosterone biosynthesis and the role on CAI [5-7]. This aroused renewed interest in aldosterone-related pharmacology in cardiovascular diseases and also aroused the interest of nephrologists to transform the ideas on kidney diseases, especially transplanted kidneys [8].

Different examinations supposed that aldosterone is involved in development and/or progression in renal diseases via interaction with a non-epithelial MR $[9,10]$. In different animal models, blockade of MR reduced for example neointima formation or renal ischemia reperfusion injury in transplantation [11-13].

Therefore a new approach in aldosterone pharmacology is not only to block the receptor but also to inhibit the biosynthesis of aldosterone itself $[14,15]$. Recently, fadrozole (FAD) 286A (a racemate also known as FAD or CGS16949A), the R-enantiomer of FAD, a non-steroidal aromatase inhibitor, has been identified as an aldosterone biosynthesis inhibitor $[13,15]$. It was shown to reduce al- dosterone levels in rats and to (partially) prevent angiotensin-II-induced mortality, cardiac remodeling, and renal damage in transgenic human renin and angiotensinogen-overexpressing rats $[6,8,16,17]$. However, only a small amount of information exists on aldosterone syntheses inhibitors with respect to CAI [18].

The majority of the experimental studies on CAI have been conducted by using the model in the Fischer 344 to Lewis rat combination [19]. In this model we studied among others the influence of immune and non-immune mechanisms of chronic graft loss, the contribution of ischemia/reperfusion injury on CAI and also the initial parenchymal injury caused by cold ischemia as the main responsible factors for chronic tubulointerstitial damage [19-21]. Fischer 344 and Lewis rats are two inbred strains which are considered haploidentical and differ only at minor histocompatibility (non-MHC) loci. These suppositions led us to examine the effects of FAD and the MR antagonist spironolactone (Spiro) compared to vehicle (VEH) in a well-established rat model of chronic transplant dysfunction.

\section{Materials and Methods}

Kidneys of male Fisher 344 rats (RT1lvl) (170-210 g) (Charles River, Sulzfeld, Germany) were transplanted into male Lewis rats (RT11) (170-210 g) (Charles River). The left native recipient kidney was removed and the left donor kidney was orthotopically transplanted by end-to-end anastomosis of renal artery, vein, and ureter under inhalative anesthesia with isoflurane. All animals received cyclosporin A subcutaneously $(1.5 \mathrm{mg} / \mathrm{kg} /$ day; SigmaAldrich, Taufkirchen, Germany) from day 0 to 10 after transplantation, when the remaining right kidney was removed. Animals received no other immunosuppressive drugs.

Initially 8 animals were assigned to each medication group and 6 animals to the control group. However, in some groups, animals died due to early posttransplantation reasons before medication application. In the end we assigned the animals to five groups: 5 animals were assigned to the AT1 group (AT1 receptor antagonist candesartan (Cand), $5 \mathrm{mg} / \mathrm{kg} /$ day); 8 were assigned to the MR antagonist group (MR antagonist Spiro $100 \mathrm{mg} / \mathrm{kg} /$ day); 6 were assigned to the combination therapy group of Cand $(5 \mathrm{mg} /$ $\mathrm{kg} /$ day) and MR antagonist (MR antagonist SPIRO $100 \mathrm{mg} / \mathrm{kg} /$ day), and 5 were assigned to the FAD group (MR antagonist $5 \mathrm{mg} /$ $\mathrm{kg} /$ day); the control group with 6 animals received $0.9 \% \mathrm{NaCl}$ (VEH). Dosage was chosen referring to the experiments in reducing cardiac fibrosis [6]. Medication was administrated orally by daily gavage.

We evaluated the effects of FAD on plasma aldosterone levels as well as urinary aldosterone excretion rate and compared their effectiveness versus the MR antagonist Spiro. The different treatments were applied for long-term treatment from day -7 to week 24 after transplantation. The observation ended after 24 weeks. In the respective groups, administration of the substances was 
initiated 7 days before transplantation in donors and recipients to achieve the maximum pharmacodynamic effect at transplantation.

Serum creatinine levels were analyzed by an automatic laboratory analyzer (Synchron CX5; Beckman Coulter, Krefeld, Germany). Mean arterial blood pressure (MAP) was measured intraarterially before the removal of the graft by a digital analyzer using a pressure transducer (Siemens, Munich, Germany). Measurement was performed under standard conditions at $24^{\circ} \mathrm{C}$ room temperature between 09:00 and 12:00 h.

\section{Morphological Studies}

Paraffin-embedded tissue sections were stained with hematoxylin and eosin (HE) to evaluate tubulointerstitial damage. CAI was graded according to parameters adapted from the Banff '97 classification: $0=$ no signs of CAI; grade $1=$ mild CAI (mild fibrosis and tubular atrophy, $5-15 \%$ of section); grade $2=$ moderate CAI (moderate fibrosis and tubular atrophy, $16-50 \%$ of section), and grade 3 = severe CAI (severe fibrosis and tubular atrophy, $>50 \%$ of section). Grading for tubulointerstitial damage included also vasculopathy with mild vasculopathy (intimal proliferation with luminal obstruction $<25 \%$ ), moderate vasculopathy (luminal obstruction $>25-50 \%$ ), and severe vasculopathy (luminal obstruction $>50 \%$ of section). Glomerulosclerosis was analyzed by counting sclerotic and normal glomeruli of the section and given as the percentage of sclerotic glomeruli.

Macrophage infiltration was evaluated with the alkaline phosphatase anti-alkaline phosphatase complex (Dako, Glostrup, Denmark) after incubation of cryostat sections $(4 \mathrm{~mm})$ with mouse anti-CD68 (Serotec, Wiesbaden, Germany) and secondary rabbit anti-mouse IgG followed by development with Fast Red chromogene solution (Dako). Cells with positive staining were counted and infiltration was graded: $0=<5,1=6-25,2=26-50$, and $3=450$ cells/field of view. At least 20 fields of view per section and per specimen were evaluated at $400 \times$ magnification.

\section{Statistical Analysis}

Data are expressed as mean \pm SEM. Data were tested using the $\chi^{2}$ test or Mann-Whitney U test. Bonferroni's method was applied to avoid inflation of type I error in multiple testing settings. A global p value of $<0.05$ was considered significant. All tests were performed two-tailed. Data were analyzed using the SPSS version 15.0 (SPSS GmbH, Munich, Germany).

\section{Results}

\section{Proteinuria after 24 Weeks of Transplantation}

Transplanted animals receiving VEH demonstrated a stable proteinuria up to 16 weeks after transplantation. Thereafter a significant rise until week 24 in proteinuria was detected $(\mathrm{p}<0.01)$. However, single treatment of the MR blocker Spiro or the aldosterone biosynthesis inhibitor FAD did not reduce proteinuria as well compared to VEH during this time period. Moreover, FAD- and Spiro-treated animals demonstrated a significantly higher proteinuria value as compared to VEH at week 24 (p <

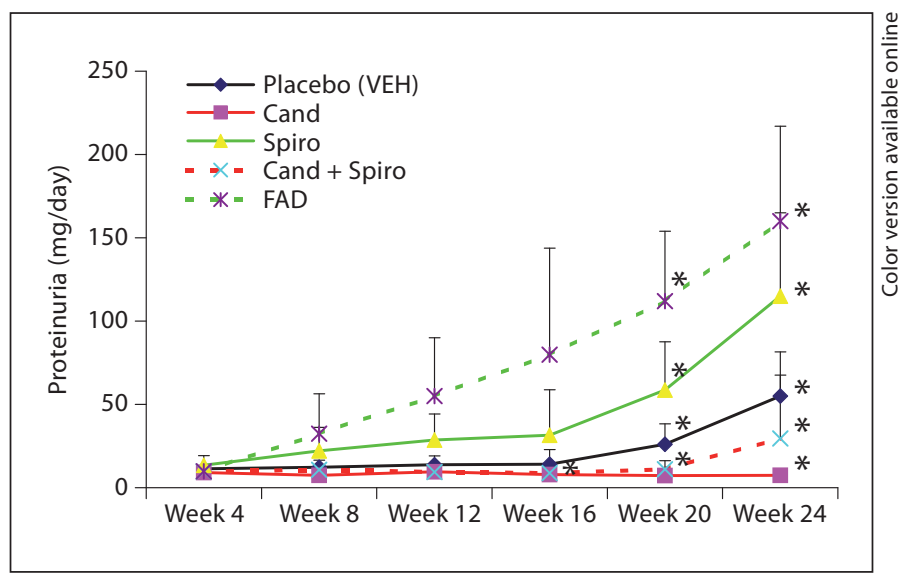

Fig. 1. Effect on proteinuria using MR antagonism and aldosterone synthesis inhibition from week 4 to week 24 with the corresponding medication. Values represent standard variation and statistically significant values are indicated $\left({ }^{*} \mathrm{p}<0.01\right)$.

0.01). Especially FAD- and Spiro-treated animals present a progradient proteinuria starting from week 8 and 12 weeks after transplantation, respectively, which accelerates explicitly from week 20 to 24 (fig. 1).

Animals receiving long-term angiotensin receptor blockade with Cand and combined Cand/Spiro treatment demonstrated less proteinuria throughout the observation, reaching significance $(\mathrm{p}<0.01)$ starting from week 16 until week 24 of observation compared to VEH and FAD or Spiro. This might be the reason why an overlap of proteinuria could be documented in figure 1 in week 24 in the Cand/Spiro and the Spiro group. One reason could be the observed tubulointerstitial damage and accelerated glomerulosclerosis, especially in the Spiro and FAD groups.

Sodium, Potassium and Serum Creatinine Levels after 24 Weeks of Transplantation

All groups irrespective of treatment presented stable serum sodium levels within the physiological range (sodium 135-145 mmol/l). Serum potassium was lowest in the Cand group. In contrast, Spiro-, FAD- and Cand/Spiro-treated animals showed higher potassium values than VEH-treated rats. In the case of Spiro- and FAD-treated animals, potassium levels were significantly pathologically elevated $(\mathrm{p}<0.05)$. With respect to $24 \mathrm{~h}$ urinary sodium excretion, all groups showed comparable values except for FAD-treated rats which demonstrated a reduced urinary sodium concentration. This pattern was identical for urinary potassium excretion (table 1). 
Table 1. Effect using MR antagonism and aldosterone synthesis inhibition referring to the basic data in serum and urine with the corresponding medication, including mortality rates

\begin{tabular}{|c|c|c|c|c|c|}
\hline & $\mathrm{VEH}$ & Spiro & FAD & Cand & Cand + Spiro \\
\hline Na serum, mmol/l & $139 \pm 2.2$ & $141 \pm 2.8$ & $143 \pm 1.3$ & $138 \pm 5.3$ & $136 \pm 18.5$ \\
\hline $\mathrm{K}$ serum, mmol/l & $5.0 \pm 0.3$ & $6.9 \pm 3.5$ & $5.6 \pm 0.6$ & $4.7 \pm 0.5$ & $5.3 \pm 0.4$ \\
\hline Na urine, $\mathrm{nmol} / \mathrm{l}$ & $0.5 \pm 0.2$ & $0.6 \pm 0.3$ & $0.2 \pm 0.1$ & $0.4 \pm 0.1$ & $0.4 \pm 0.1$ \\
\hline $\mathrm{K}$ urine, $\mathrm{nmol} / \mathrm{l}$ & $1.9 \pm 0.3$ & $1.8 \pm 0.4$ & $1.3 \pm 0.1$ & $2.1 \pm 1.0$ & $1.8 \pm 0.2$ \\
\hline Aldosterone urine, $\mathrm{nmol} / \mathrm{l}$ & $0.4 \pm 0.4$ & $0.5 \pm 1.0$ & $0.2 \pm 0.1$ & $0.2 \pm 0.2$ & $0.4 \pm 1.0$ \\
\hline \multicolumn{6}{|l|}{ Mortality rates } \\
\hline until week 24 & $\begin{array}{l}\text { all animals lived } \\
\text { until week } 24\end{array}$ & $\begin{array}{l}\text { all animals lived } \\
\text { until week } 24\end{array}$ & $\begin{array}{l}3 \text { animals died at week } \\
16,2 \text { at week } 24\end{array}$ & $\begin{array}{l}\text { all animals lived } \\
\text { until week } 24\end{array}$ & $\begin{array}{l}\text { all animals lived } \\
\text { until week } 24\end{array}$ \\
\hline
\end{tabular}

Statistically significant values are highlighted in bold (ANOVA with post hoc Dunnett vs. vehicle group; Spiro and FAD p < 0.05).

Table 2. Effect using MR antagonism and aldosterone synthesis inhibition referring to the basic data with the corresponding medication, including the histological grading of tubulointerstitial damage and glomerulosclerosis

\begin{tabular}{|c|c|c|c|c|c|}
\hline & VEH & Spiro & FAD & Cand & Cand + Spiro \\
\hline BW, g & $458.2 \pm 48$ & $401.3 \pm 90$ & $366.8 \pm 103$ & $478.7 \pm 9$ & $454.3 \pm 24.1$ \\
\hline $\mathrm{KW} / \mathrm{BW}, \mathrm{g} / \mathrm{kg}$ & $3.6 \pm 0.2$ & $4.2 \pm 0.5$ & $4.0 \pm 0.7$ & $4.2 \pm 0.3$ & $4.1 \pm 0.1$ \\
\hline $\mathrm{MAP}, \mathrm{mm} \mathrm{Hg}$ & $103.0 \pm 3.3$ & $99.5 \pm 4.6$ & $92.6 \pm 2.5$ & $85.1 \pm 2.7$ & $89.4 \pm 2.4$ \\
\hline Tubolointerstitial & & $\mathrm{p}<0.01$ & $\mathrm{p}<0.05$ & $\mathrm{p}<0.05$ & \\
\hline \multicolumn{6}{|l|}{ Damage } \\
\hline Grade 1 & 4 & 2 & 1 & 1 & 3 \\
\hline Grade 2 & 2 & 2 & 3 & 2 & 3 \\
\hline Grade 3 & 0 & 4 & 1 & 0 & 0 \\
\hline \multicolumn{6}{|c|}{ CD68 macrophage infiltration } \\
\hline cells/field of view & $3.3 \pm 1.5$ & $5.8 \pm 2.6$ & $4.8 \pm 1.8$ & $1.3 \pm 0.6$ & $3.0 \pm 1.9$ \\
\hline Glomerulosclerosis, \% & $68.9 \pm 10.2$ & $63.9 \pm 7.2$ & $98.2 \pm 1.8$ & $45.9 \pm 4.3$ & $67.9 \pm 6.7$ \\
\hline
\end{tabular}

Statistically significant values for glomerulosclerosis are highlighted in bold (ANOVA with post hoc Dunnett vs. VEH group; Cand $\mathrm{p}<0.05$; FAD $\mathrm{p}<0.01$ ). Significance for tubulointerstitial damage is also included (Spiro $\mathrm{p}<0.01, \mathrm{FAD} \mathrm{p}<0.05$, Cand $\mathrm{p}<0.05$ ).

Serum creatinine rates were stable in the VEH, Cand and Cand/Spiro groups. Related to the elevated potassium levels, creatinine parameters were also significantly higher in the FAD and Spiro groups $(\mathrm{p}<0.05)$.

\section{Aldosterone Levels after 24 Weeks of Transplantation}

Cand in monotherapy or in combination with Spiro had no effect on the serum aldosterone concentration. In contrast, Spiro monotherapy resulted in markedly elevated serum aldosterone, whereas the aldosterone synthase inhibitor FAD resulted in reduced aldosterone concentration. Urinary aldosterone concentration was comparable in VEH- and Spiro- or Cand/Spiro-treated animals, but tended to a non-significantly reduced level in Candor FAD-treated animals (table 1).

\section{Body Weight after 24 Weeks of Transplantation}

Throughout the groups, body weight (BW) was comparable except for FAD-treated animals which developed a BW lower than VEH-treated rats (table 2). Animals 
treated with Spiro and Cand/Spiro tended to develop also a lower BW than animals with Cand antagonist or VEH treatment. VEH-treated animals tended to a smaller kidney weight/body weight ratio (KW/BW) as compared to all other treatment groups. With respect to cardiac hypertrophy, Cand-treated rats tended to a lower heart weight/body weight (HW/BW) as VEH-treated rats, whereas Spiro- and FAD-treated rats showed a markedly increased HW/BW.

\section{Blood Pressure after 24 Weeks of Transplantation}

MAP was highest in the VEH group (table 2). Cand-, Cand/Spiro- and FAD-treated animals had a lower MAP compared to VEH, whereas Spiro-treated animals had a similar MAP as VEH rats.

Grade of CAI CTD after 24 Weeks of Transplantation

VEH animals developed mild tubulointerstitial damage which was significantly $(\mathrm{p}<0.05)$ less intense in Cand-treated rats, whereas FAD- $(\mathrm{p}<0.05)$ and Spirotreated $(p<0.01)$ animals tended to have enhanced and significantly more tubulointerstitial damage compared to VEH and Cand, respectively. Cand/Spiro was comparable to VEH but not statistically significant. The same pattern was observed with respect to CD68-positive macrophages infiltrating the tubulointerstitium (table 2). However, differences in CD68 infiltration did not reach significance.

The glomerular pattern showed a high number of sclerotic glomeruli in the VEH group. Glomerulosclerosis was significantly $(\mathrm{p}<0.05)$ less prominent after Cand treatment. Spiro or the combination Cand/Spiro had no influence on the amount of sclerotic glomeruli. However, FAD-treated animals had a significantly $(\mathrm{p}<0.01)$ enhanced number of sclerotic changes as compared to VEH. In fact, nearly all glomeruli were sclerotic. The extended sclerotic lesions were associated with the fact that 3 animals died until week 16.

Interstitial fibrosis was ameliorated in the Cand group compared to VEH. Spiro or FAD had no positive influence on the amount of interstitial fibrosis. The combination of Cand/Spiro showed the highest grade of interstitial fibrosis.

\section{Discussion}

This study demonstrates that in our model of CAI, neither a direct MR blockade nor a biosynthesis inhibitor had a positive effect on renal CAI in rats. On the contrary,
FAD- and Spiro-treated animals demonstrated a higher proteinuria value, significantly and pathologically elevated potassium values, higher tubulointerstitial damage fibrosis and markedly increased HW/BW as compared to VEH.

In some cardiac models the usage of aldosterone receptor antagonists showed their ability to prevent and reverse cardiac fibrosis and led to the supposition of reduction of interstitial fibrosis in transplanted kidneys $[13,16$, 17]. In previous examinations it was detected that aldosterone also had an influence on fibroblast activation and the production of collagen in rat renal fibroblasts. It was suggested that a blockade of the aldosterone pathway has a positive impact on interstitial fibrosis as detected in some cardiac models [13, 24].

In the present study, the direct MR blockade presents the effects as expected: Spiro monotherapy resulted in markedly elevated serum aldosterone, whereas the urinary aldosterone concentration tended to have a non-significantly reduced level in Spiro-treated animals. Both are typical characteristics of the feedback mechanism after blockade of the MR [5].

In our examination a receptor blockade of aldosterone with Spiro had no positive effect on tubulointerstitial damage fibrosis. The supposed positive effects could not be confirmed in Spiro-treated transplanted kidneys compared to VEH (fig. 2a, b) $[6,19,23]$. On the other hand, several animals treated with Spiro demonstrated severe tubulointerstitial damage. This finding was not present in glomeruli, therefore suggesting that the damage in this group was predominantly presented in the tubulointerstitial area. As Spiro leads to an augmented aldosterone level due to a positive feedback loop, MR-independent mechanisms could be involved in the damage [9]. In this context, elevated potassium levels augment rat renal MR selectivity via $11 \beta$-hydroxysteroid dehydrogenase [24]. As Spiro-treated rats had an elevated potassium level, this influence on the salt-water homeostasis may have further augmented tubulointerstitial damage. Also, a combination therapy of Cand and Spiro presented no beneficial effect on glomerulosclerosis or tubulointerstitial damage fibrosis.

In the present study the direct aldosterone biosynthesis inhibitor also presents an effective treatment as expected: the usage of FAD resulted in a reduced serum aldosterone concentration, whereas the urinary aldosterone concentration tended to non-significantly reduce the level in FAD-treated animals. As opposed to Spirotreated animals, no augmented tubulointerstitial damage was present and potassium levels remained normal in 

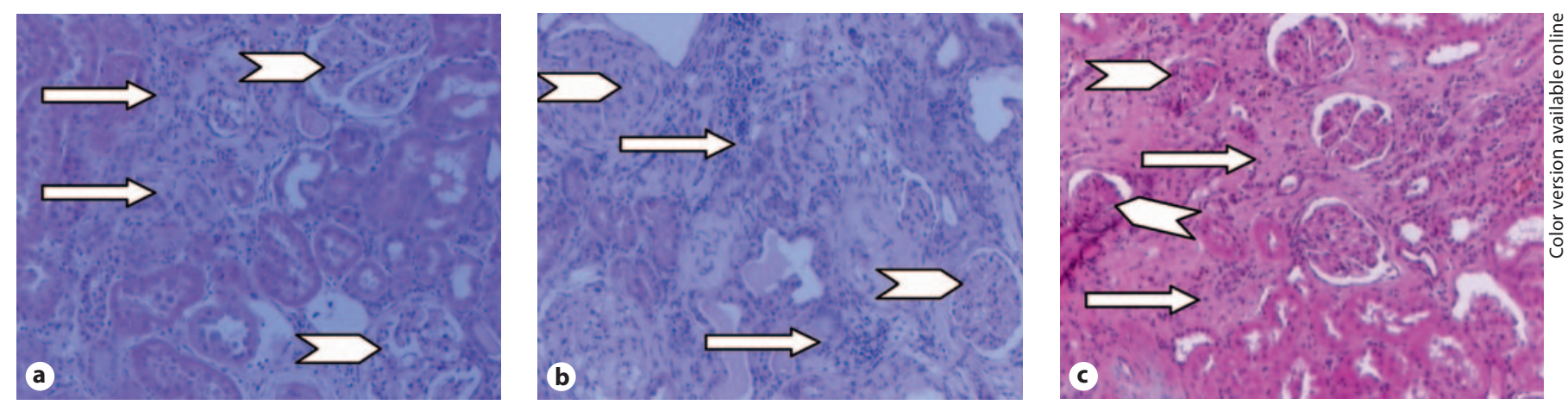

Fig. 2. Histological effect using MR antagonism and aldosterone synthesis inhibition. a A representative image of $\mathrm{VEH}$ with HE staining presenting glomerulosclerosis (short arrows) and interstitial fibrosis (long arrows). b, c HE staining with glomerulosclerosis (short arrows) and interstitial fibrosis (long arrows).

FAD-treated animals. However, severe glomerulosclerosis was observed in these animals involving nearly all glomeruli (fig. 2c). This stands in contrast to the finding of the effective inhibition of aldosterone biosynthesis by FAD. As the idea of beneficial effects of reduced aldosterone level has been repetitively demonstrated, this finding is unexpected. The histological sclerosis was associated with an enhanced number of transplanted rats dying during the second month of FAD treatment. We therefore suggest a mechanism which may be rather associated by drug effects. Indeed, the pharmacokinetics of FAD could explain these results $[25,26]$. After oral administration a rapid, completely absorbed (mean bioavailability of 99.9\%) and extensive distribution to tissue could be detected $[14,26]$. Approximately $60 \%$ are bound to plasma proteins, mainly to albumin. The major route of elimination is metabolism by CYP-450 in the liver into an inactive carbinol metabolite $[14,26]$. Renal impairment is not described with an increased $\mathrm{T}_{1 / 2}$ as seen in a hepatic impairment, but there may be toxic metabolites that can lead to a fast glomerulosclerosis as observed in our model. However, there are no data about transplanted rats and renal impairment, or if the inactive metabolites interact with for example immunosuppressant or impaired kidney function $[15,26]$. Further examinations therefore have to be made with regard to interactions of pharmacokinetics in kidney recipients. A small effect could be seen in FAD-treated animals, respectively to the blood pressure. They had a lower MAP compared to VEH, whereas Spiro-treated animals showed a similar MAP as $\mathrm{VEH}$ rats. The reduced blood pressure in FAD-treated animals can be explained by the reduced aldosterone biosynthesis [13].
Another observation could be made of the heart and body weight after transplantation. Spiro- and FAD-treated rats showed a markedly increased HW/BW ratio caused by a renal-heart feedback mechanism of the reninangiotensin-aldosterone system blockade, which is linked to reduced organ survival. This is in line with the protective effects of both drugs in cardiac hypertrophy and fibrosis, suggesting a small cardio-beneficial effect after all.

\section{Conclusion}

To conclude, our study demonstrates that at the moment a positive effect of Spiro or FAD on renal CAI in rats could not be detected. Especially the higher rates of tubulointerstitial damage in Spiro and glomerulosclerosis in FAD-treated rats can lead to severe side effects in kidney recipients. Further examinations had to be made to use the positive effects of direct or indirect inhibition of aldosterone for kidney recipients as described in cardiac models.

\section{Disclosure Statement}

The authors have no conflicts of interest to disclose. 


\section{References}

1 Chapman JR, O'Connell PJ, Nankivell BJ: Chronic renal allograft dysfunction. J Am Soc Nephrol 2005; 16:3015-3026.

-2 Campbell PM, Cascalho M, Collins AB, et al: Banff '05 Meeting Report: differential diagnosis of chronic allograft injury and elimination of chronic allograft nephropathy ('CAN'). Am J Transplant 2007;7:518-526.

- 3 Cosio FG, Pelletier RP, Sedmak DD, Falkenhain ME, Henry ML, Elkhammas EA, Davies EA, Bumgardner GL, Ferguson RM: Pathologic classification of chronic allograft nephropathy: pathogenic and prognostic implications. Transplantation 1999;67:690696.

4 Lutz J, Risch K, Liu S, Antus B, Schmaderer C, Roos M, Ouyang N, Lehmann M, Heemann U: Angiotensin type 1 and type 2 receptor blockade in chronic allograft nephropathy. Kidney Int 2006;70:1080-1088.

$\checkmark 5$ Macdonald JE, Kennedy N, Struthers AD: Effects of spironolactone on endothelial function, vascular angiotensin converting enzyme activity, and other prognostic markers in patients with mild heart failure already taking optimal treatment. Heart 2004;90: 765-770.

6 Cohn JN, Colucci W: Cardiovascular effects of aldosterone and post-acute myocardial infarction pathophysiology. Am J Cardiol 2006;97:4F-12F.

7 Ward MR, Kanellakis P, Ramsey D, Funder J, Bobik A: Eplerenone suppresses constrictive remodeling and collagen accumulation after angioplasty in porcine coronary arteries. Circulation 2001;104:467-472.

-8 Del Vecchio L, Procaccio M, Vigano S, Cusi D: Mechanisms of disease: the role of aldosterone in kidney damage and clinical benefits of its blockade. Nat Clin Pract Nephrol 2007;3:42-49.

$\checkmark 9$ Rocha R, Chander PN, Zuckerman A, Stier CT Jr: Role of aldosterone in renal vascular injury in stroke-prone hypertensive rats. Hypertension 1999;33:232-237.
10 Caprio M, Newfell BG, la SA, Baur W, Fabbri A, Rosano G, Mendelsohn ME, Jaffe IZ: Functional mineralocorticoid receptors in human vascular endothelial cells regulate intercellular adhesion molecule-1 expression and promote leukocyte adhesion. Circ Res 2008;102:1359-1367.

-11 Aldigier JC, Kanjanbuch T, Ma LJ, Brown NJ, Fogo AB: Regression of existing glomerulosclerosis by inhibition of aldosterone. J Am Soc Nephrol 2005;16:3306-3314.

12 Wakabayashi K, Suzuki H, Sato T, Iso Y, Katagiri T, Takeyama Y: Eplerenone suppresses neointimal formation after coronary stent implantation in swine. Int J Cardiol 2006;107:260-266.

13 Minnaard-Huiban M, Emmen JMA, Roumen L, Beugels IPE, et al: Fadrozole reverses cardiac fibrosis in spontaneously hypertensive heart failure rats: discordant enantioselectivity versus reduction of plasma aldosterone. Endocrinology 2008;149:28-31.

14 Stein RC, Dowsett M, Davenport J, Hedley A, Ford HT, Gazet JC, Coombes RC: Preliminary study of the treatment of advanced breast cancer in postmenopausal women with the aromatase inhibitor CGS16949A. Cancer Res 1990;50:1381-1384.

15 Menard J, Gonzalez MF, Guyene TT, Bissery: A Investigation of aldosterone-synthase inhibition in rats. J Hypertens 2006;24:11471155.

16 Fiebeler A, Muller DN, Shagdarsuren E, Luft FC: Aldosterone, mineralocorticoid receptors, and vascular inflammation. Curr Opin Nephrol Hypertens 2007;16:134-142.

17 Remuzzi G, Cattaneo D, Perico N: The aggravating mechanisms of aldosterone on kidney fibrosis. J Am Soc Nephrol 2008;19: 1459-1462.

18 Mejia-Vilet JM, Ramirez V, Cruz C, Uribe N, Gamba G, Bobadilla NA: Renal ischemia-reperfusion injury is prevented by the mineralocorticoid receptor blocker spironolactone. Am J Physiol Renal Physiol 2007; 293:F78-F86.
19 Bedi DS, Riella LV, Tullius SG, Chandraker A: Animal models of chronic allograft injury: contributions and limitations to understanding the mechanism of long-term graft dysfunction. Transplantation 2010;90:935944

20 Tullius SG, Reutzel-Selke A, Egermann F, et al: Contribution of prolonged ischemia and donor age to chronic renal allograft dysfunction. J Am Soc Nephrol 2000;11:1317-1324.

-21 Herrero-Fresneda I, Torras J, Cruzado JM, et al: Do alloreactivity and prolonged cold ischemia cause different elementary lesions in chronic allograft nephropathy? Am J Pathol 2003;162:127-137.

22 Lea WB, Kwak ES, Luther JM, Fowler SM, Wang Z, Ma J, Fogo AB, Brown NJ: Aldosterone antagonism or synthase inhibition reduces end-organ damage induced by treatment with angiotensin and high salt. Kidney Int 2009;75:936-944.

23 Connell JMC, Davies E: The new biology of aldosterone. J Endocrinol 2005;186:1-20.

24 Hermans JJ, Fischer MA, Schiffers PM, Struijker-Boudier HA: High dietary potassium chloride intake augments rat renal mineralocorticoid receptor selectivity via $11 \beta$-hydroxysteroid dehydrogenase. Biochim Biophys Acta 1999;1472:537-549.

25 Garnier A, Bendall JK, Fuchs S, Escoubet B, Rochais F, Hoerter J, Nehme J, Ambroisine ML, De Angelis N, Morineau G, d'Estienne P, Fischmeister R, Heymes C, Pinet F, Delcayre C: Cardiac specific increase in aldosterone production induces coronary dysfunction in aldosterone synthase-transgenic mice. Circulation 2004;110:1819-1825.

26 LaSala D, Shibanaka Y, Jeng AY: Coexpression of CYP11B2 or CYP11B1 with adrenodoxin and adrenodoxin reductase for assessing the potency and selectivity of aldosterone synthase inhibitors. Anal Biochem 2009;394: $56-61$. 